\title{
La competencia digital del profesorado de literatura en Educación Secundaria en España
}

\author{
A competência digital dos professores de literatura no ensino \\ médio na Espanha \\ The digital competence of secondary school literature teachers in Spain
}

\author{
Francisco José Rodríguez Muñoz (D) *1 y María del Mar \\ Ruiz-Domínguez (D) †1
}

${ }^{1}$ Universidad de Almería, Departamento de Educación, Almería, Andalucía, España.

\begin{abstract}
Resumen
Esta investigación parte del interés de conocer la competencia digital del profesorado de Educación Secundaria que enseña literatura en España. Dada la naturaleza multidimensional de esta competencia, el presente estudio busca responder específicamente al grado de conocimiento y uso de los recursos educativos TIC en el aula de literatura. Para ello, se diseñó un cuestionario que cumplimentaron 71 docentes en activo de la especialidad de Lengua Castellana y Literatura adscritos a centros educativos españoles. El análisis estadístico de los datos (descriptivo, $t$-Student, chi-cuadrado y $r$ de Pearson) reveló información significativa sobre aspectos tales como el bajo porcentaje de profesorado con intención de formarse en TIC, las relaciones entre el uso de recursos TIC y las variables género y edad, por un lado, y titularidad y ubicación de los centros, por otro, así como sobre el grado de utilidad que los docentes de Secundaria perciben al trabajar con recursos educativos TIC concretos y al valorar su aprovechamiento en relación con distintos elementos de la educación literaria.
\end{abstract}

Palabras clave: Desarrollo profesional docente. Educación literaria. Formación del profesorado. Recursos TIC.

\section{Resumo}

Esta pesquisa se baseia no interesse em conhecer a competência digital de professores de literatura do ensino médio na Espanha. Dada a natureza multidimensional dessa competência, este estudo procura responder especificamente ao grau de conhecimento e utilização dos recursos TIC na aula de literatura. Para isso, foi elaborado e respondido um questionário por 71 professores ativos da especialidade de Língua e Literatura Espanholas, lotados em centros educacionais espanhóis. A análise estatística dos dados (descritiva, $t$-Student, chi-quadrado e $r$ de Pearson) revelou informações significativas sobre aspectos como a baixa percentagem de professores com intenção de formação em TIC, as relações entre a utilização de recursos TIC e as variáveis gênero e idade, por um lado, e propriedade e localização dos centros, por outro, bem como o grau de utilidade

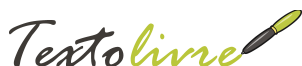

Linguagem e Tecnologia

DOI: $10.35699 / 1983-$

3652.2021.31351

Sección:

Artículos

Autor correspondiente:

Francisco José Rodríguez

Muñoz

Editor de sección:

Hugo Heredia Ponce

Editor de maquetación:

Daniervelin Pereira

Recibido el:

9 de marzo de 2021

Aceptado el:

18 de abril de 2021

Publicado el:

13 de agosto de 2021

Esta obra está bajo una licencia «CC BY 4.0». ()ㅜ) que os professores do ensino médio percebem ao trabalhar com recursos educacionais específicos de TIC e ao avaliar seu uso em relação a diferentes elementos da educação literária.

Palavras-chave: Desenvolvimento profissional de professores. Educação literária. Treinamento de professor. Recursos TIC.

\section{Abstract}

This research arises from the interest of knowing the digital competence of secondary school teachers who teach literature in Spain. Given the multidimensional nature of this competence, the study seeks to look specifically at the level of knowledge and the use of ICT educational resources in the literature classroom. To do this, a questionnaire was designed and completed by 71 active teachers from Spanish schools, whose specialty is Spanish Language and Literature. The statistical data analysis (descriptive, Student's t-test, chi-squared and Pearson's r) revealed significant information regarding, on the one hand, aspects such as the low percentage of teachers who intend to train themselves in ICT, the relationships between the use of ICT resources and the gender and age variables; and on the other, the types of educational centres and their locations as well as how useful secondary school teachers consider working with specific ICT educational resources. Their use of ICT educational resources in relation to the different elements of literary education were also assessed.

Keywords: Teacher professional development. Literary education. Teacher training. ICT resources.

\footnotetext{
*Email: frodriguez@ual.es

†Email: mmruiz@ual.es
} 


\section{Introducción}

Atendiendo al contexto digital en el que nos desenvolvemos en la vida diaria y a los imparables procesos de digitalización que exigen las mil y una actividades de tipo personal y social en las que estamos implicados, la actual legislación española en materia educativa, la Ley Orgánica 3/2020 (ESPAÑA, 2020), reconoce que esta dependencia de las TIC conlleva un cambio más que evidente en la comprensión de la realidad circundante y en las formas de participar en esta:

Este cambio de enfoque requiere de una comprensión integral del impacto personal y social de la tecnología, de cómo este impacto es diferente en las mujeres y los hombres y una reflexión ética acerca de la relación entre tecnologías, personas, economía y medioambiente, que se desarrolle tanto en la competencia digital del alumnado como en la competencia digital docente. En consecuencia, se hace necesario que el sistema educativo dé respuesta a esta realidad social e incluya un enfoque de la competencia digital más moderno y amplio, acorde con las recomendaciones europeas relativas a las competencias clave para el aprendizaje permanente (ESPAÑA, 2020, p. 122871).

La complejidad que supone el dominio de la competencia digital (en el caso que aquí nos ocupa, del profesorado) se aprecia, por ejemplo, en los esfuerzos por discriminar los componentes que la conforman. Vuorikari et al. (2016) señalan las cinco subcompetencias siguientes: (1) la información y alfabetización informacional, (2) la comunicación y colaboración, (3) la creación de contenidos digitales, (4) la seguridad, y (5) la resolución de problemas. En este sentido, y en el ámbito de la formación docente, el Marco europeo para la competencia digital de los educadores (DigCompEdu) (REDECKER y PUNIE, 2017) hace hincapié en la relevancia de algunas de estas subcompetencias al insistir en el compromiso profesional que ha de contraerse por parte de los docentes con el uso de las TIC aplicadas a los procesos de enseñanza-aprendizaje. Asimismo, destaca la implicación activa que se consigue cuando estos recursos son empleados en clase por el alumnado, ya que asisten al fomento de la creatividad, la reflexividad y la responsabilidad, y le permiten informarse, comunicarse, crear contenido y resolver problemas.

Por lo tanto, actualmente resulta impensable que el profesorado ignore este panorama tecnológico, y su nivel competencial en TIC se ha convertido en un tema de estudio prioritario desde hace ya algún tiempo, debido a las consecuencias que entraña para el desarrollo educativo (SUÁREZ RODRÍGUEZ; ALMERICH y col., 2012; LÁZARO-CANTABRANA; USART-RODRÍGUEZ y GISBERT-CERVERA, 2019; CABERO-ALMENARA y PALACIOS-RODRÍGUEZ, 2020; ORTIZ COLÓN; ÁGREDA MONTORO y RODRÍGUEZ MORENO, 2020). Más aún, la situación de pandemia ocasionada por la COVID-19 ha constatado como nunca la dependencia de los docentes, en cualquier etapa educativa, a los medios tecnológicos para dar continuidad a la enseñanza en modalidad virtual o combinada con la presencial. Al mismo tiempo, ha puesto al descubierto que, a pesar de que existan diferentes recursos en los centros, o de que estos se encuentren más o menos actualizados, los docentes han de contar con un nivel en competencia digital que les permita adaptarse y responder de manera rápida, efectiva y poco traumática a los procesos de digitalización y virtualización que exigen circunstancias como la referida (GÓMEZ JIMÉNEZ; RODRÍGUEZ TORRES y CRUZ CRUZ, 2020; SÁ y SERPA, 2020).

El dominio de la competencia digital del profesorado estaría definido por el mayor o mejor dominio de las subcompetencias referidas al conocimiento de los recursos TIC, a su diseño e implementación, así como al análisis y la valoración de los recursos en el uso docente (DURÁN CUARTERO; GUTIÉRREZ PORLÁN y PRENDES ESPINOSA, 2016). En este sentido, el modelo de competencia digital docente propuesto en DigCompEdu (REDECKER y PUNIE, 2017) está estructurado en cinco áreas, entre las que se encuentran las que tienen que ver con la selección, creación, uso y distribución de contenidos digitales en el aula (área 2) y la relacionada con la implementación de los recursos digitales en el proceso de enseñanza-aprendizaje (área 3). Se trata de que los docentes integren las TIC en el diseño y el desarrollo curricular; lo que precisa poseer unos conocimientos y una formación que les permitan, 
entre otras cosas, saber escoger aquellos recursos educativos que sean significativos y que puedan vincularse a la realización de tareas complejas. No obstante, la formación específica en TIC para su uso en el aula a menudo presenta carencias (ALMERICH; SUÁREZ-RODRÍGUEZ y col., 2011); comprobar esta información constituirá uno de los propósitos del presente trabajo.

En esta investigación, nos centramos en las competencias digitales docentes que se incluyen en las áreas competenciales 2 y 3 de DigCompEdu (REDECKER y PUNIE, 2017), es decir, las relacionadas con el dominio y la aplicación didáctica de potenciales recursos educativos TIC para el aula de literatura como son los textos literarios para tableta, textos literarios transmedia, fanfiction, Wattpad, Goodreads, BookTube, literatura en tráileres, foros de lectura literaria, revistas digitales de literatura y digital storytelling, entre otros. No consideramos, por consiguiente, aquellas herramientas digitales que están al servicio del docente y que son de consulta o de preparación de sus clases. Tampoco hemos dado cabida en esta investigación a las herramientas que, con la llegada de la COVID-19, se han implementado para la docencia virtual, puesto que estos recursos no se justifican por los contenidos de las materias, sino por la necesidad de reproducir el contexto del aula en un entorno virtual.

¿Realmente se han incorporado en España recursos como los fanfictions o BookTube a las aulas de literatura? ¿Tienen los profesores suficiente información y formación para utilizarlos? ¿La falta de formación, el desconocimiento y la baja percepción de su utilidad podrían ser motivos de su baja presencia o de su escaso uso en el aula? De estas preguntas emana el objetivo general de la investigación: descubrir el grado de conocimiento, uso y aceptación de los recursos digitales en el aula de literatura en la etapa de Educación Secundaria. Este objetivo general se ha concretado en cuatro específicos:

1. Conocer si el profesorado español de Secundaria ha recibido formación sobre recursos educativos TIC para aplicarlos en sus clases de literatura.

2. Conocer la frecuencia de uso de recursos educativos TIC en las clases de literatura y su aprovechamiento en el aula.

3. Identificar los recursos educativos TIC que se utilizan en el aula de literatura en Educación Secundaria.

4. Descubrir el grado de aceptación de recursos educativos TIC en clase de literatura.

Con carácter previo al diseño del cuestionario a partir del cual se recogieron los datos que se presentan en este trabajo, se llevó a cabo una revisión bibliográfica que permitió distinguir tres grupos de investigaciones: (1) estudios sobre la aplicación de recursos TIC en el aula de literatura (p. ej., Mula-Franco, Llorens-García y Rovira-Collado (2010), Jwa (2012), Rovira-Collado y Hernández Ortega (2016), Valero Gómez y Granados Pérez (2016) y Miranda Lacerda y Schlemmer (2018)); (2) análisis estadísticos para conocer la incidencia de las redes sociales en el desarrollo de las competencias lectoras (p. ej., Rovira Collado (2015), Amiama-Espaillat y Mayor-Ruiz (2017) y Torres (2018); y (3) trabajos sobre las actitudes y las afinidades de los estudiantes respecto a los textos literarios digitales (p. ej., (CENTRO REGIONAL PARA EL FOMENTO DEL LIBRO EN AMÉRICA LATINA Y EL CARIBE (BOGOTÁ) y AGENCIA ESPAÑOLA DE COOPERACIÓN INTERNACIONAL PARA EL DESARROLLO, 2014; SCOLARI, 2018)).

El rastreo bibliográfico permitió corroborar que no se habían planteado con anterioridad investigaciones específicamente encaminadas a descubrir el grado de utilización y aceptación de los recursos TIC para el aula de literatura por parte del profesorado de Educación Secundaria. No obstante, se acudió a distintos trabajos publicados en los que se cuantifica de manera general la competencia digital del profesorado de diferentes niveles educativos (ALMERICH; GASTALDO y col., 2004; PRENDESESPINOSA; CASTAÑEDA-QUINTERO y GUTIÉRREZ-PORLÁN, 2010; SUÁREZ RODRÍGUEZ; ALMERICH CERVERÓ y col., 2010; DOMÍNGUEZ ALFONSO, 2011; SÁNCHEZ PRIETO; OLMOS MIGUELÁÑEZ y GARCÍA-PEÑALVO, 2015; NIX y HALL, 2016).

Por ello, una vez analizados los trabajos que describen la intención de uso ante las TIC del profesorado (SÁNCHEZ PRIETO; OLMOS MIGUELÁÑEZ y GARCÍA-PEÑALVO, 2015; NIX y HALL, 2016), y para dar respuesta a nuestros objetivos de investigación, establecimos una serie de hipótesis que se irán detallando en los párrafos sucesivos.

En primer término, consideramos que existe una relación directa entre formación en recursos TIC 
y edad. Es decir, pensamos que, aunque el profesorado, por lo general, no suele tener formación específica en herramientas TIC para aplicarlas en el aula de literatura, las generaciones más jóvenes de profesorado de literatura estarían más preparadas que las de mayor edad por pertenecer a una generación digital que les ha proporcionado acceso a estos recursos de una manera más natural e intuitiva. Por el contrario, consideramos que no existirán diferencias en cuanto a formación entre profesores y profesoras, esto es, en función del género. Nuestras hipótesis serían, por lo tanto, las siguientes:

H1 Los profesores de Secundaria tienen, en general, escasa formación en recursos educativos TIC para aplicar en las clases de literatura.

H2 Los profesores más jóvenes tienen más formación que los de más edad.

H3 Las profesoras tienen la misma formación que los profesores.

En segundo término, respecto a la frecuencia de uso, esta se encontraría directamente relacionada con la formación previa y el conocimiento de estos recursos por parte de los docentes, con la utilidad percibida y con la familiaridad y uso de herramientas digitales en el ámbito privado del profesorado. En este sentido, pensamos que los profesores más jóvenes, tanto hombres como mujeres, utilizarán más a menudo los recursos TIC en el aula de literatura que los de mayor edad. En consecuencia, formulamos cinco hipótesis:

H4 Los profesores más jóvenes utilizan las TIC en su vida diaria con más frecuencia.

H5 Los profesores más jóvenes utilizan más recursos educativos TIC en sus clases de literatura que los de mayor edad.

H6 Las profesoras utilizan los recursos TIC con la misma frecuencia en el aula que sus compañeros de género masculino.

H7 Los profesores más jóvenes creen que los recursos educativos TIC son útiles para sus clases, frente a los de mayor edad.

H8 Existe una correlación entre los profesores que utilizan pocos recursos digitales en su vida diaria y la baja utilización de recursos TIC en sus clases de literatura.

En tercer término, en cuanto al uso que se les da a los recursos TIC en el aula, creemos que suelen implementarse para profundizar en conceptos teóricos, para mostrar autores y sus obras, así como para promover la lectura en clase. Además, creemos que los recursos más utilizados son los más conocidos, es decir, los textos literarios digitales, los foros literarios de webs de literatura y las revistas literarias en línea:

H9 Los recursos educativos TIC se utilizan sobre todo para presentar contenidos y actividades acerca de autores y obras literarias, tipologías textuales y lectura de clásicos.

H10 Los recursos que más se utilizan son textos literarios para tableta, foros literarios y revistas digitales de literatura.

Por último, intuimos que la ubicación del centro y la titularidad determinan una mayor o menor aceptación de estas herramientas:

H11 Los profesores cuyos centros de enseñanza radican en núcleos urbanos utilizan recursos educativos TIC con mayor frecuencia que los profesores que trabajan en zonas más alejadas de los centros urbanos.

H12 Los profesores de centros privados-concertados, en general, perciben que sus estudiantes muestran un mayor dominio de los recursos educativos TIC que los profesores de centros públicos.

\section{Metodología}

\subsection{Participantes}

El perfil que se exigía a los participantes en esta investigación tenía que responder a las siguientes características: profesorado de Educación Secundaria de la especialidad de Lengua Castellana y Literatura que se encontrara en activo en el momento de la recogida de datos, adscrito indistintamente a centros públicos o privados/concertados ubicados en España.

De los 125 participantes que respondieron inicialmente al cuestionario solo se consideraron 71 , que fueron quienes siguieron las instrucciones, contestando todas las preguntas e informando de las variables sociodemográficas que se les solicitaban. Del total de informantes que respondieron a nuestras 
preguntas, un $76,1 \%$ fueron mujeres y un $23,9 \%$ hombres. Las edades estuvieron comprendidas entre los 20 y los 60 años, con un promedio de 39,9 \pm 9,9 años. El 84,5\% desempeñaba su actividad docente en centros públicos, mientras que el $15,5 \%$ lo hacía en centros privados o concertados. El promedio de experiencia docente que correspondió a la muestra fue de 12 años (mín.-máx.: 1-34, DT = 9,5). En relación con la distribución geográfica de los centros de enseñanza del profesorado participante, el 53,5 $\%$ se localizaba en capitales de provincia y el $46,5 \%$ en pueblos. Más específicamente, colaboraron docentes de veintitrés centros de la provincia de Granada, quince de la provincia de Almería, seis de Madrid, cuatro de Málaga, cuatro de Sevilla, cuatro de Valencia, dos de Cádiz, dos de Castellón de la Plana y uno procedente de las provincias de Ávila, Cáceres, Ciudad Real, Jaén, La Coruña, Lérida, Mallorca, Murcia, Valladolid y Zamora.

\subsection{Instrumento}

El instrumento de medida empleado para la recogida de datos fue el cuestionario "Conocimiento, uso y aceptación de recursos educativos TIC en clase de literatura". Tras diseñar una primera versión de este, que fue sometida a un juicio de especialistas $(n=6)$, la fase de revisión resultó en la mejora de la propuesta de ítems tanto en forma como en contenido. Una vez depurado el cuestionario, se aplicó a una muestra de cinco participantes con objeto de identificar y eliminar posibles errores. Después de efectuar dicho pilotaje, el cuestionario fue dividido en dos secciones: (1) datos sociodemográficos (género, edad, años en activo, tipo de centro y localidad en la que este radica), y (2) veintiuna preguntas integradas por setenta ítems formulados de distinta manera: en escala Likert de 5 o 6 puntos, en preguntas cerradas, en preguntas de respuesta múltiple y en preguntas de respuesta abierta. Asimismo, se determinaron siete dimensiones en torno a las que se agruparon los ítems sin repartirlos linealmente, a saber: (1) uso personal, (2) grado de conocimiento, (3) uso real, (4) utilidad percibida, (5) dificultades percibidas, (6) intención conductual, y (7) percepción del uso y dominio de los estudiantes.

Finalmente, se calculó la fiabilidad del cuestionario por medio del alfa de Cronbach, cuyos valores fueron siempre superiores a 0,8 en cada una de las dimensiones, así como el coeficiente de correlación lineal de Pearson (RUIZ-DOMÍNGUEZ y RODRÍGUEZ-MUÑOZ, 2021).

\subsection{Procedimiento}

El cuestionario fue creado y difundido, principalmente, mediante la herramienta de encuestas en línea LimeSurvey, a la que nos dio acceso el Servicio de las Tecnologías de la Información y las Comunicaciones (STIC) de la Universidad de Almería (España). Por lo tanto, el cuestionario pudo cumplimentarse a través de cualquier dispositivo con conexión a internet entre el 5 de febrero y el 1 de abril de 2019. No obstante, antes de cerrar la fase recogida de datos, se distribuyeron varios cuestionarios en papel y se incorporaron manualmente las respuestas correspondientes a nueve de ellos en la plataforma LimeSurvey.

El promedio de tiempo que se tardó en contestar todas las preguntas del cuestionario fue de 9,7 minutos, por lo que se avino con la advertencia inicial en la que se pronosticaba una duración aproximada de 10 minutos para cumplimentarlo.

\subsection{Análisis estadístico}

El tratamiento estadístico de los datos fue realizado con el programa SPSS para Windows (versión 25). El análisis no solo incluye los estadísticos descriptivos, con porcentajes y medias, correspondientes a las respuestas que proporcionaron los participantes, sino que también se ejecutaron análisis comparativos mediante las siguientes pruebas: $t$-Student, chi-cuadrado y $r$ de Pearson. Estas permitieron determinar la significación estadística de variables como la formación recibida, el tipo de centro, la ubicación de este, el género y la edad de los participantes. 


\section{Resultados y discusión}

3.1 Grado de conocimiento, intención conductual y dificultades percibidas sobre recursos educativos TIC en literatura

En la Tabla 1 se muestra el descriptivo correspondiente a las preguntas de elección única del cuestionario en las que se relacionan las dimensiones "Grado de conocimiento", "Intención conductual" y "Dificultades percibidas". Según estos resultados, están bastante equiparados los porcentajes de docentes de Educación Secundaria que han recibido o están recibiendo formación sobre recursos TIC destinados a las clases de literatura, así como los relativos a la participación en redes sociales y comunidades virtuales relevantes para su uso en el aula de literatura. En cambio, contrasta el bajo porcentaje de profesores que tienen la intención de seguir formándose en los próximos meses, frente al elevado número de enseñantes que indican que su centro dispone de los medios para su uso. Los porcentajes de estas dos preguntas contrastan, ya que no muestran interés en continuar formándose, a pesar de poder aplicarlos en el aula.

Si bien, como se ha indicado, la mayor parte del profesorado considera que su centro dispone de los medios necesarios para utilizar recursos educativos TIC, en los centros en los que la infraestructura tecnológica no es suficiente o adecuada, opina que, de serlo, los emplearía en clase.

Tabla 1. Descriptivo correspondiente a las preguntas de elección única sobre grado de conocimiento, intención conductual y dificultades percibidas.

\begin{tabular}{|c|c|c|c|}
\hline Dimensión & Pregunta & $\mathrm{n}$ & $\%$ \\
\hline & $\begin{array}{l}\text { ¿Ha recibido o está recibiendo formación específica sobre re- } \\
\text { cursos educativos TIC relacionados con la literatura? }\end{array}$ & & \\
\hline & No & 42 & 59,2 \\
\hline \multirow[t]{4}{*}{ Grado de conocimiento } & Sí & 29 & 40,8 \\
\hline & $\begin{array}{l}\text { ¿Participa en redes sociales o comunidades virtuales en las } \\
\text { que se ofrecen contenidos e información sobre recursos edu- } \\
\text { cativos TIC para su uso en clase de literatura? }\end{array}$ & & \\
\hline & No & 29 & 40,8 \\
\hline & Sí & 42 & 59,2 \\
\hline \multirow[t]{4}{*}{ Intención conductual } & $\begin{array}{l}\text { ¿Tiene previsto realizar algún curso de formación en recur- } \\
\text { sos educativos TIC relacionados con la literatura durante los } \\
\text { próximos } 6 \text { meses? }\end{array}$ & & \\
\hline & No & 54 & 76,1 \\
\hline & Sí & 17 & 23,9 \\
\hline & $\begin{array}{l}\text { ¿Dispone su centro educativo de los medios necesarios que } \\
\text { le permiten utilizar recursos educativos TIC relacionados con } \\
\text { la literatura? }\end{array}$ & & \\
\hline \multirow[t]{5}{*}{ Dificultades percibidas } & No & 18 & 25,4 \\
\hline & Sí & 53 & 74,6 \\
\hline & $\begin{array}{l}\text { Si su centro contase con los medios necesarios, ¿utilizaría } \\
\text { recursos educativos TIC en sus clases de literatura? }\end{array}$ & & \\
\hline & No & 1 & 5,6 \\
\hline & Sí & 17 & 94,4 \\
\hline
\end{tabular}

Fuente: Elaboración propia.

\subsection{Puntuaciones según la formación recibida}

Para establecer la relación existente entre las dimensiones de la escala con la formación recibida sobre recursos educativos TIC, hemos procedido a realizar la prueba t-Student. Como refleja la Tabla 2, los resultados evidenciaron que el nivel de conocimiento de los docentes de Secundaria que no han recibido formación $(3,31)$ fue significativamente superior al de los que sí la han recibido $(1,53)$. Se 
cumple, por lo tanto, nuestra primera hipótesis referida a la baja utilización de estos recursos (H1).

Habría que indagar las causas que subyacen a estos resultados, los cuales podrían ser indicativos, por ejemplo, de que los profesores que no han recibido formación, y, sin embargo, poseen más conocimientos sobre recursos TIC de literatura, consideran que no la necesitan, o bien pueden ser sintomáticos de que dicha formación es deficiente o está mal planteada, entre otras posibles razones. En el resto de las dimensiones, no se observaron diferencias estadísticamente significativas entre los docentes según la formación recibida.

Tabla 2. Descriptivo y comparativo de las puntuaciones en cada una de las dimensiones del cuestionario según formación.

\begin{tabular}{|c|c|c|c|c|c|}
\hline \multirow[t]{2}{*}{ Dimensión } & \multicolumn{2}{|c|}{ Formación TIC } & \multirow{2}{*}{$\begin{array}{l}\text { Diferencia } \\
\text { medias }\end{array}$} & \multicolumn{2}{|c|}{ Prueba t-Student } \\
\hline & No & Sí & & $t(g . l)$. & p-valor \\
\hline Uso personal & $3,49(0,79)$ & $3,72(0,73)$ & $-0,23$ & $\mathrm{t}(69)=-1,215$ & 0,229 \\
\hline Grado conocimiento & $3,31(0,51)$ & $1,53(0,56)$ & 1,78 & $t(28)=3,116$ & 0,004 \\
\hline Uso real & $0,75(0,63)$ & $0,81(0,61)$ & $-0,06$ & $t(69)=-0,411$ & 0,682 \\
\hline Utilidad percibida & $2,23(0,6)$ & $2,21(0,67)$ & 0,02 & $t(55)=0,116$ & 0,908 \\
\hline Dificultades percibidas & $2,48(0,76)$ & $2,55(0,91)$ & $-0,07$ & $t(69)=-0,381$ & 0,704 \\
\hline Intención conductual & $0,54(0,26)$ & $0,67(0,26)$ & $-0,13$ & $t(16)=-0,97$ & 0,346 \\
\hline Percepción uso estudiantes & $2,96(0,86)$ & $2,77(0,71)$ & 0,19 & $t(69)=0,984$ & 0,328 \\
\hline
\end{tabular}

Fuente: Elaboración propia.

Por otra parte, en la Tabla 3 se muestra el descriptivo de las respuestas a las preguntas relacionadas con utilidad de la formación recibida y la participación en contextos informales, y su relación con el género del informante, así como los resultados de las pruebas chi-cuadrado para determinar la posible relación entre estas respuestas y el género. Como se aprecia en dicha tabla, el porcentaje de docentes que no han recibido formación continua y que participan en redes sociales $(47,6 \%)$ fue significativamente inferior al de los que sí la han recibido $(75,9 \%$ ) y participan en dichas redes o comunidades en las que se ofrecen contenidos para su uso en el aula de literatura. Esto evidencia que las carencias formativas de estos docentes son suplidas por contextos y entornos de formación no oficiales.

3.3 Relaciones del género y la edad con las dimensiones de la escala

En la Tabla 4 se muestra el descriptivo de las puntuaciones de las dimensiones de la escala según el género, así como los resultados de las pruebas t-Student realizadas para comparar las puntuaciones entre mujeres y hombres. Las pruebas confirman que el grado de conocimiento de las mujeres $(M=$ $1,4)$ es significativamente inferior al de los hombres $(M=2,12)$ que imparten clase de literatura en Secundaria. En el resto de las dimensiones no se observaron diferencias estadísticamente significativas entre mujeres y hombres. Podríamos pensar que, si las mujeres tienen menor grado de conocimiento, debieran hacer menor uso real de estas herramientas en el aula. Sin embargo, las medias indican que poseen un menor grado de conocimiento, pero no son significativas en cuanto a "Uso real", lo que quiere decir que utilizan las TIC tanto como los hombres, pero sin contar con tanta formación o conocimiento sobre ellas. Estos resultados implican el cumplimiento de la hipótesis referida al mismo uso de estas herramientas entre hombres y mujeres (H6), pero no ratifican la equiparación en la formación entre profesores y profesoras ( $\mathrm{H} 2)$.

Para determinar la posible relación entre las puntuaciones de las dimensiones de la escala con la edad, se calculó el coeficiente de correlación lineal de Pearson ( $r$ ), cuyos resultados se muestran en la Tabla 5. La edad mostró una correlación estadísticamente significativa y negativa con el "Uso personal", el "Uso real" y la "Utilidad percibida", de forma que, a mayor edad, menor "Uso personal", menor "Uso real" y menor "Utilidad percibida". En este sentido, los informantes de mayor edad no han recibido ni tienen intención de recibir formación, y no están de acuerdo con que los recursos TIC 
Tabla 3. Descriptivo y comparativo de las puntuaciones en cada una de las dimensiones del cuestionario según formación.

\begin{tabular}{|c|c|c|c|c|}
\hline \multirow[t]{2}{*}{ Pregunta } & \multicolumn{2}{|c|}{ Formación TIC } & \multicolumn{2}{|c|}{ Prueba chi-cuadrado } \\
\hline & No & Sí & $2(\mathrm{~g} . \mathrm{l})$. & p-valor \\
\hline $\begin{array}{l}\text { La formación recibida sobre recursos educati- } \\
\text { vos TIC para trabajar la literatura en el aula } \\
\text { me ha resultado sido útil }\end{array}$ & & & $2(3)=0,599$ & 0,897 \\
\hline Totalmente en desacuerdo & $0(0)$ & $0(0)$ & & \\
\hline Algo en desacuerdo & $0(0)$ & $3(10,3)$ & & \\
\hline Indiferente & $0(0)$ & $2(6,9)$ & & \\
\hline Algo de acuerdo & $1(100)$ & $18(62,1)$ & & \\
\hline Totalmente de acuerdo & $0(0)$ & $6(20,7)$ & & \\
\hline $\begin{array}{l}\text { ¿Participa en redes sociales o comunidades } \\
\text { virtuales en las que se ofrecen contenidos e in- } \\
\text { formación sobre recursos educativos TIC para } \\
\text { su uso en clase de literatura? }\end{array}$ & & & $2(1)=5,663$ & 0,017 \\
\hline No & $22(52,4)$ & $7(24,1)$ & & \\
\hline Sí & $20(47,6)$ & $22(75,9)$ & & \\
\hline
\end{tabular}

Fuente: Elaboración propia.

Tabla 4. Descriptivo y comparativo de las puntuaciones en cada una de las dimensiones del cuestionario según el género.

\begin{tabular}{llllll}
\hline \multirow{2}{*}{ Dimensión } & \multicolumn{2}{l}{ Género, media (DT) } & $\begin{array}{l}\text { Diferencia } \\
\text { medias }\end{array}$ & Prueba t-Student \\
& Femenino & Masculino & & $\mathrm{t}(\mathrm{g} . \mathrm{l})$. & p-valor \\
\hline Uso personal & $3,56(0,72)$ & $3,67(0,93)$ & $-0,11$ & $\mathrm{t}(69)=-0,504$ & 0,616 \\
Grado conocimiento & $1,4(0,5)$ & $2,12(0,72)$ & $-0,72$ & $\mathrm{t}(28)=-3,075$ & $\mathbf{0 , 0 0 5}$ \\
Uso real & $0,71(0,59)$ & $0,97(0,69)$ & $-0,26$ & $\mathrm{t}(69)=-1,548$ & 0,126 \\
Utilidad percibida & $2,19(0,64)$ & $2,28(0,6)$ & $-0,09$ & $\mathrm{t}(55)=-0,435$ & 0,665 \\
Dificultades percibidas & $2,4(0,81)$ & $2,85(0,76)$ & $-0,45$ & $\mathrm{t}(69)=-2,012$ & 0,048 \\
Intención conductual & $0,57(0,26)$ & $0,67(0,29)$ & $-0,1$ & $\mathrm{t}(16)=-0,603$ & 0,555 \\
Percepción uso estudiantes & $2,93(0,84)$ & $2,74(0,7)$ & 0,19 & $\mathrm{t}(69)=0,851$ & 0,398 \\
\hline
\end{tabular}

Fuente: Elaboración propia. 
sirvan para mejorar la docencia. En consecuencia, se cumplen las hipótesis en las que se cruza la edad del profesorado con el uso personal en la vida diaria y con el uso real en el aula puntos $\mathrm{H} 4, \mathrm{H} 5$ y $\mathrm{H} 8$, así como con la utilidad percibida punto $\mathrm{H} 7$, pero no la referida a la formación y al conocimiento Punto $\mathrm{H} 2$.

Tabla 5. Coeficiente de correlación de Pearson entre la edad y las dimensiones de la escala.

\begin{tabular}{ll}
\cline { 2 - 2 } Dimensión & Edad, r (p-valor) \\
\cline { 2 - 3 } Uso personal & $-0,323(0,006)$ \\
Grado conocimiento & $0,055(0,774)$ \\
Uso real & $-0,339(0,045)$ \\
Utilidad percibida &,$- 0321(0,015)$ \\
Dificultades percibidas & $-0,018(0,881)$ \\
Intención conductual & $-0,366(0,135)$ \\
Percepción uso estudiantes & $-0,076(0,528)$ \\
\hline
\end{tabular}

Fuente: Elaboración propia.

3.4 Utilidad percibida de los recursos educativos TIC sobre literatura y cursos en los que se utilizan

Si se atiende al grado de utilidad, como muestra la Figura 1, la mayoría de los recursos educativos TIC incluidos en el cuestionario coinciden en ser considerados bastante útiles por parte de los profesores de Secundaria que imparten clase de literatura, salvo Wattpad y Goodreads, que se valoran como algo útiles, tal vez por tratarse de plataformas menos conocidas que las otras. De todos estos recursos, más de la mitad de los docentes han considerado que los textos literarios para tableta, los textos transmedia y los foros de lectura literaria son "bastante" o "muy" útiles. Se cumpliría, por ello, nuestra hipótesis H10, aunque es cierto que, a juicio de los participantes, no existen grandes diferencias en cuanto a la mayor o menor utilidad según el tipo de recursos.

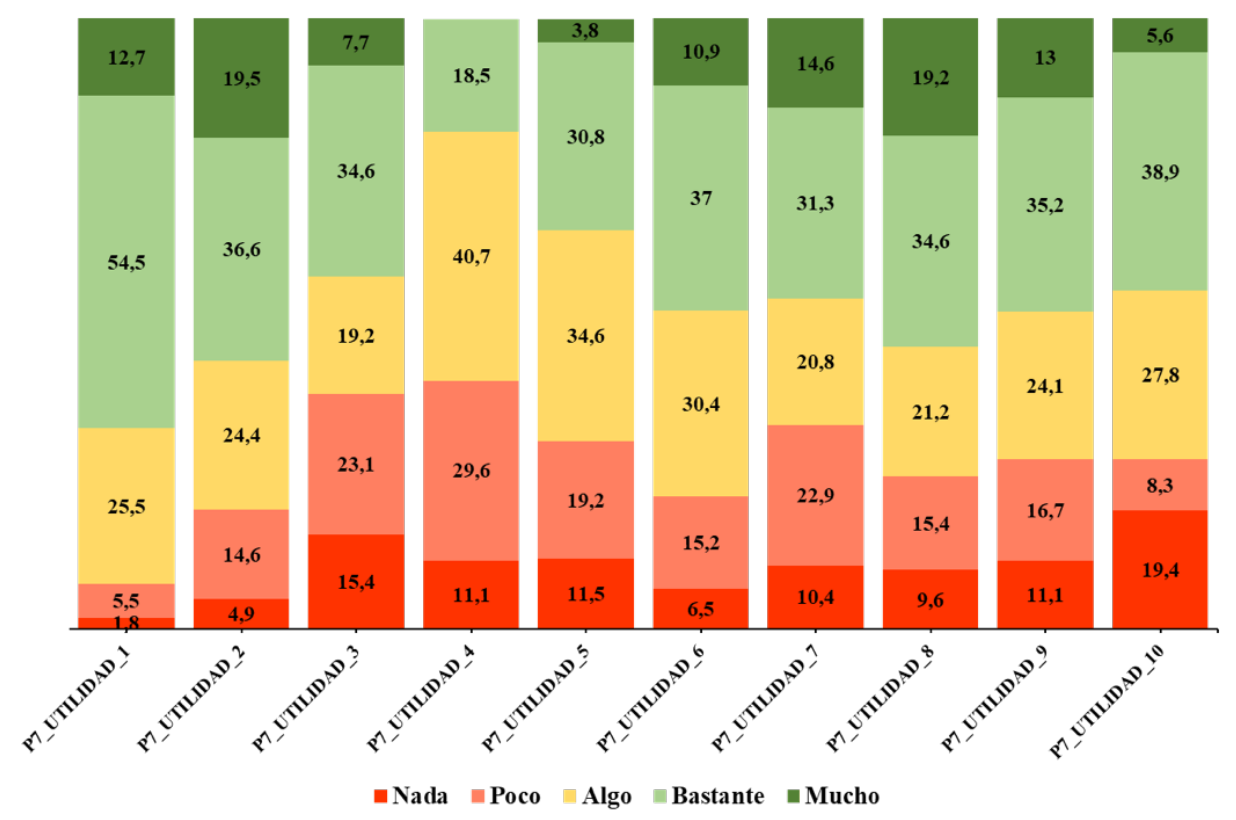

Figura 1. Porcentajes relativos al grado de utilidad de los recursos educativos TIC sobre literatura incluidos en el cuestionario.

Fuente: Elaboración propia.

Nota: P7_1 = Textos literarios para tableta; P7_2 = Textos literarios transmedia; P7_3 = Fanfiction; P7_4 = Wattpad; P7_5 = Goodreads; PT_6 = BookTube; P7_7 = Literatura en tráileres; P7_8 = Foros de lectura literaria; P7_9 = Revistas digitales de literatura; P7_10 = Digital storytelling. 
Tras preguntarle al profesorado de Secundaria que imparte literatura en qué medida ha contribuido de manera positiva la utilización de algún recurso TIC al desarrollo de los elementos incluidos en la Tabla 6, se observa que tales recursos educativos han resultado muy útiles al trabajar "contenidos y actividades sobre elementos narratológicos", así como "actividades de animación a la lectura". En los demás casos, los recursos TIC han sido bastante útiles, opinión que también predominaba en la valoración particular de cada uno (Figura 1), y tan solo se consideran algo útiles al ponerse en relación con la "comprensión lectora". No obstante, todas las dimensiones han sido valoradas de manera muy similar, por lo que no podemos establecer con claridad los fines para los que se utilizan los recursos TIC en el aula.

Tabla 6. Porcentajes correspondientes al grado de utilidad de los recursos educativos TIC en relación con distintos elementos asociados a la educación literaria.

\begin{tabular}{|c|c|c|c|c|c|}
\hline \multirow{2}{*}{$\begin{array}{l}\text { Elementos asociados a la educación lite- } \\
\text { raria }\end{array}$} & \multicolumn{5}{|c|}{ Respuesta, n (\%) } \\
\hline & Nada & Poco & Algo & Bastante & Mucho \\
\hline $\begin{array}{l}\text { Contenidos y actividades sobre autores y } \\
\text { obras literarias }\end{array}$ & $2(3,2)$ & $0(0)$ & $15(23,8)$ & $28(44,4)$ & $18(28,6)$ \\
\hline $\begin{array}{l}\text { Contenidos y actividades sobre elemen- } \\
\text { tos narratológicos }\end{array}$ & $11(18,3)$ & $4(6,7)$ & $15(25)$ & $13(21,7)$ & $17(28,3)$ \\
\hline $\begin{array}{l}\text { Contenidos y actividades sobre tipologías } \\
\text { textuales y géneros literarios }\end{array}$ & $7(11,3)$ & $2(3,2)$ & $16(25,8)$ & $22(35,5)$ & $15(24,2)$ \\
\hline Comprensión lectora & $6(10)$ & $2(3,3)$ & $19(31,7)$ & $16(26,7)$ & $17(28,3)$ \\
\hline Capacidad crítica & $5(8,3)$ & $3(5)$ & $17(28,3)$ & $20(33,3)$ & $15(25)$ \\
\hline Actividades de animación a la lectura & $5(8,2)$ & $4(6,6)$ & $10(16,4)$ & $17(27,9)$ & $25(41)$ \\
\hline Actividades de escritura creativa & $9(14,8)$ & $3(4,9)$ & $13(21,3)$ & $21(34,4)$ & $15(24,6)$ \\
\hline $\begin{array}{l}\text { Lectura de textos clásicos de literatura } \\
\text { española o universal }\end{array}$ & $6(10,2)$ & $2(3,4)$ & $14(23,7)$ & $21(35,6)$ & $16(27,1)$ \\
\hline $\begin{array}{l}\text { Lectura de textos de literatura infantil y } \\
\text { juvenil }\end{array}$ & $8(13,3)$ & $5(8,3)$ & $13(21,7)$ & $19(31,7)$ & $15(25)$ \\
\hline
\end{tabular}

Fuente: Elaboración propia.

Por último, de acuerdo con los resultados, el curso en el que el profesorado participante ha empleado recursos educativos TIC con mayor frecuencia en sus clases de literatura ha sido $4 .^{\circ}$ de ESO (62 \%), seguido muy de cerca por los cursos de $2 .^{\circ}$ de $\operatorname{ESO}(57,7 \%), 3 .^{\circ}$ de $\operatorname{ESO}(56,3 \%)$ y $4 . .^{\circ}$ de $\operatorname{ESO}(53,5$ $\%)$. Los cursos en los que menos se utilizan recursos TIC son $1 .^{\circ}$ de Bachillerato $(42,3 \%)$ y $2 .^{\circ}$ de Bachillerato $(31 \%)$. Si bien sería conveniente indagar las causas que hay detrás de estos porcentajes, parece plausible que no sea tan habitual el uso de recursos TIC en 2. ${ }^{\circ}$ de Bachillerato por ser este un curso orientado a la preparación de la Prueba de Evaluación de Bachillerato para el Acceso a la Universidad (PEvAU), lo que implica una mayor rigidez en la planificación; asimismo, pueden influir otras variables como la edad del profesorado que a menudo se ocupa de la docencia en estos cursos, entre otras.

\subsection{Puntuaciones según la titularidad del centro y su ubicación}

Con relación al tipo de centro, como muestra la Tabla 7, se observaron diferencias estadísticamente significativas en las dimensiones "Uso personal" y "Percepción uso estudiantes" entre los docentes de los centros públicos y los de los centros privados/concertados. Estos resultados informan de que los profesores que trabajan en estos últimos centros usan en su vida diaria las TIC con mayor frecuencia que los pertenecientes a centros públicos de enseñanza. Asimismo, los docentes de centros privado$\mathrm{s} /$ concertados afirman que sus estudiantes se sitúan levemente por encima de los de centro públicos en cuanto a dominio de recursos TIC, uso de estos para su formación literaria, etc.

En la Tabla 8 se muestran los estadísticos descriptivos y los resultados de las pruebas en función de la ubicación de los centros escolares. No se advirtieron diferencias estadísticamente significativas 
Tabla 7. Porcentajes correspondientes al grado de utilidad de los recursos educativos TIC en relación con distintos elementos asociados a la educación literaria.

\begin{tabular}{llllll}
\hline Dimensión & \multicolumn{2}{c}{ Titularidad del centro } & $\begin{array}{l}\text { Diferencia } \\
\text { medias }\end{array}$ & Prueba t-Student & \\
& Público & $\begin{array}{l}\text { Privado/ } \\
\text { concertado }\end{array}$ & & $\mathrm{t}(\mathrm{g} . \mathrm{l})$. & p-valor \\
\hline Uso personal & $3,5(0,74)$ & $4,05(0,8)$ & $-0,55$ & $\mathrm{t}(69)=-2,203$ & 0,031 \\
Grado conocimiento & $1,56(0,55)$ & $1,83(1,14)$ & $-0,27$ & $\mathrm{t}(28)=-0,784$ & 0,44 \\
Uso real & $0,75(0,57)$ & $0,89(0,86)$ & $-0,14$ & $\mathrm{t}(69)=-0,702$ & 0,485 \\
Utilidad percibida & $2,19(0,61)$ & $2,34(0,74)$ & $-0,15$ & $\mathrm{t}(55)=-0,619$ & 0,538 \\
Dificultades percibidas & $2,52(0,81)$ & $2,44(0,91)$ & 0,08 & $\mathrm{t}(69)=0,31$ & 0,757 \\
Intención conductual & $0,61(0,29)$ & $0,5(0)$ & 0,11 & $\mathrm{t}(16)=0,724$ & 0,479 \\
Percepción uso estudiantes & $2,8(0,76)$ & $3,3(0,93)$ & $-0,5$ & $\mathrm{t}(69)=-2,012$ & 0,048 \\
\hline
\end{tabular}

Fuente: Elaboración propia.

en ninguna de las dimensiones entre los centros ubicados en las capitales de provincia y los que tienen una ubicación más marginal. En consecuencia, no se han podido demostrar nuestras hipótesis ??.

Tabla 8. Descriptivo y comparativo de las puntuaciones en cada una de las dimensiones del cuestionario según ubicación.

\begin{tabular}{llllll}
\hline \multirow{2}{*}{ Dimensión } & \multicolumn{2}{l}{ Capital provincia } & $\begin{array}{l}\text { Diferencia } \\
\text { medias }\end{array}$ & Prueba t-Student \\
& No & Sí & & $\mathrm{t}(\mathrm{g} . \mathrm{l})$. & p-valor \\
\hline Uso personal & $3,61(0,66)$ & $3,56(0,87)$ & 0,05 & $\mathrm{t}(69)=0,276$ & 0,783 \\
Grado conocimiento & $1,54(0,47)$ & $1,62(0,74)$ & $-0,08$ & $\mathrm{t}(28)=-0,327$ & 0,746 \\
Uso real & $0,83(0,65)$ & $0,72(0,59)$ & 0,11 & $\mathrm{t}(69)=0,759$ & 0,45 \\
Utilidad percibida & $2,19(0,69)$ & $2,25(0,56)$ & $-0,06$ & $\mathrm{t}(55)=-0,36$ & 0,72 \\
Dificultades percibidas & $2,54(0,77)$ & $2,48(0,86)$ & 0,06 & $\mathrm{t}(69)=0,309$ & 0,758 \\
Intención conductual & $0,75(0,29)$ & $0,54(0,24)$ & 0,21 & $\mathrm{t}(16)=1,526$ & 0,147 \\
Percepción uso estudiantes & $2,89(0,78)$ & $2,87(0,84)$ & 0,02 & $\mathrm{t}(69)=0,132$ & 0,895 \\
\hline
\end{tabular}

Fuente: Elaboración propia.

\section{Conclusiones}

La presente investigación ha revelado la necesidad de plantear estudios de esta naturaleza, ya que ha puesto al descubierto la escasa o inadecuada formación que reciben los docentes en cuanto a las herramientas TIC y su aplicación en el aula, particularmente en España. Así pues, ante el desconocimiento, la percepción de la utilidad de los recursos TIC en las clases de literatura es baja, y no podremos esperar una utilización generalizada de estos.

Si bien es verdad que son recursos que, por su novedad, van incorporándose en la actualidad a las metodologías docentes, no es menos cierto que se le va otorgando cada vez más importancia al necesario dominio de la competencia TIC por parte del profesorado (ALMERICH; GASTALDO y col., 2004; PRENDES-ESPINOSA; CASTAÑEDA-QUINTERO y GUTIÉRREZ-PORLÁN, 2010; INSTITUTO NACIONAL DE TECNOLOGÍAS EDUCATIVAS Y DE FORMACIÓN DEL PROFESORADO (INTEF), 2017). En este sentido, los programas de formación continua y actualización del profesorado en materia de metodologías docentes deben ser revisados, o, al menos, cuestionados, habida cuenta de que la mayor parte del profesorado que ha participado en esta investigación ha reconocido que no cuenta con formación, o bien que esta es insuficiente, ya que muchos acuden a comunidades de aprendizaje mediante el acceso a foros y espacios de discusión en la red para adquirir conocimientos 
sobre este tipo de recursos. Aun así, más del $75 \%$ de los docentes encuestados declararon no tener previsto realizar ningún curso de formación en el futuro. Sería interesante averiguar a qué es debida dicha falta de interés; como se ha señalado anteriormente, tal vez podría explicarse por la planificación de unos cursos TIC cuyos contenidos no ofrecen respuestas a las demandas específicas del profesorado de literatura de Educación Secundaria, que acudiría a contextos informales de aprendizaje donde sería posible acceder a una formación más precisa, más actualizada o en el momento que más le conviniera.

Por otra parte, en nuestra investigación también se ha constatado que existe una relación directa entre el nivel generacional del docente y la utilización de estas herramientas en la clase de literatura. El profesorado más joven, usuario más habitual de redes sociales en su vida personal, emplea con más frecuencia estos recursos en sus clases de literatura, frente al profesorado de más edad. Son los docentes más jóvenes, conocedores de estas herramientas, los que muestran una actitud más positiva ante su uso y utilidad. Los profesores de más edad, por el contrario, muestran mayor reticencia o resistencia a la hora de usar estas herramientas, puesto que las desconocen y, por consiguiente, no perciben su utilidad.

Respecto a las diferencias en función del género, esta investigación ha revelado que las profesoras han recibido menos formación en recursos TIC que los hombres; en cambio, las distinciones según el género se igualan en la utilización de estos recursos. Profesores y profesoras aplican en la misma medida herramientas TIC en sus clases de literatura. Cabría pensar, por ello, que las profesoras han alcanzado el conocimiento de estas herramientas motu proprio, mediante la búsqueda de información. En la investigación llevada a cabo por Ortiz Colón, Ágreda Montoro y Rodríguez Moreno (2020, p. 62), se aprecia que, entre los docentes de Educación Primaria, también son los hombres quienes dan cuenta de un mayor "contenido tecnológico"; es decir, en comparación con las profesoras, estos poseerían un mayor conocimiento en cuanto "a la variedad de tecnologías y a las posibilidades de aplicación a su práctica docente".

La variable ubicación geográfica del centro en el que el docente imparte clase no ha condicionado el mayor o menor uso de herramientas TIC. Esto podría deberse a que la aplicación de recursos TIC en el aula de literatura requiere de una buena conexión a internet y de un hardware y un software actualizados, con independencia de la distancia del centro educativo respecto a grandes núcleos de población. Por otra parte, resultaría interesante indagar la relación existente entre la edad del profesorado, la situación geográfica del centro en el que imparte docencia y el mayor o menor uso de estas herramientas.

En síntesis, en este trabajo se hace patente la necesidad de reforzar la formación en competencias digitales del profesorado tanto en la formación inicial como en la permanente, así como la de revisar los contenidos que se imparten en los diferentes cursos y talleres sobre recursos TIC en el aula de literatura y su indispensable adecuación a las actuales demandas docentes. Como línea futura de investigación, sería oportuno comprobar si la obligada implementación de entornos virtuales para la docencia, provocada por la irrupción de la COVID-19, ha favorecido la formación del profesorado en competencias digitales y si los recursos TIC para trabajar contenidos de literatura se están convirtiendo en herramientas habituales en contextos presenciales de enseñanza-aprendizaje.

\section{Financiación}

Esta investigación fue realizada en el marco del proyecto La lectura en la era digital: nuevas prácticas lectoras, cultura participativa y espacios de afinidad (EDU2015-69924-R), financiado por el Ministerio de Economía y Competitividad (Programa Estatal de I+D+i Orientada a los Retos de la Sociedad), Gobierno de España.

\section{Referencias}

ALMERICH, Gonzalo; GASTALDO, I. y col. Perfiles de las competencias en las TIC y su relación con la utilización de las mismas en los profesores de Educación Primaria y Secundaria, jun. 2004. Disponible en: <http://e-spacio.uned.es/fez/view/bibliuned:19563>. Acceso en: 13 ago. 2021.

ALMERICH, Gonzalo; SUÁREZ-RODRÍGUEZ, Jesús y col. Las necesidades formativas del profesorado en TIC: Perfiles formativos y elementos de complejidad. RELIEVE - Revista Electrónica de Investigación y 
Evaluación Educativa, v. 17, n. 2, sep. 2011. DOI: 10.7203/relieve.17.2.4006. Disponible en: <https://ojs.uv.es/index.php/RELIEVE/article/view/4006>. Acceso en: 13 ago. 2021.

AMIAMA-ESPAILLAT, Cristina y MAYOR-RUIZ, Cristina. Digital reading and reading competence. The influence in the Z generation from the Dominican Republic. Comunicar, v. 25, n. 52, p. 105-114, jul. 2017. DOI: 10.3916/C52-2017-10. Disponible en:

$<$ https://www.revistacomunicar.com/index.php?contenido=detalles\&numero $=52 \&$ articulo $=52-2017-10>$. Acceso en: 13 ago. 2021.

CABERO-ALMENARA, Julio y PALACIOS-RODRíGUEZ, Antonio. Marco Europeo de Competencia Digital Docente «DigCompEdu». Traducción y adaptación del cuestionario «DigCompEdu Check-In». EDMETIC, v. 9, n. 1, p. 213-234, dic. 2020. DOI: 10.21071/edmetic.v9i1.12462. Disponible en:

<https://www.uco.es/servicios/ucopress/ojs/index.php/edmetic/article/view/12462>. Acceso en: 13 ago. 2021.

CENTRO REGIONAL PARA EL FOMENTO DEL LIBRO EN AMÉRICA LATINA Y EL CARIBE (BOGOTÁ) y AGENCIA ESPAÑOLA DE COOPERACIÓN INTERNACIONAL PARA EL DESARROLLO. Metodología común para explorar y medir el comportamiento lector: el encuentro con lo digital. Bogotá: CERLAC-UNESCO, 2014. OCLC: 913121758.

DOMíNGUEZ ALFONSO, Rocío. Formación, Competencias y Actitudes sobre las TIC del Profesorado de Secundaria: Un Instrumento de Evaluación. Etic@net: Revista científica electrónica de Educación y Comunicación en la Sociedad del Conocimiento, 10 (Junio), pág. 6, 2011. Disponible en: $<$ https://dialnet.unirioja.es/servlet/articulo?codigo=3702718>. Acceso en: 13 ago. 2021.

DURÁN CUARTERO, Marta; GUTIÉRREZ PORLÁN, Isabel y PRENDES ESPINOSA, María Paz. Propuesta de un modelo actualizado de competencia digital del profesorado: áreas, descriptores e indicadores. In: TECNOLOGÍA, innovación e investigación en los procesos de enseñanza-aprendizaje. [S.I.]: Octaedro, 2016. p. 1587-1598. Disponible en:

<https://dialnet.unirioja.es/servlet/articulo?codigo=6066204>. Acceso en: 13 ago. 2021.

ESPAÑA. Ley Orgánica 3/2020, de 29 de diciembre, por la que se modifica la Ley Orgánica 2/2006, de 3 de mayo, de Educación. [S.I.: s.n.], 2020. Disponible en:

<https://www.boe.es/eli/es/lo/2020/12/29/3/dof/spa/pdf>. Acceso en: 13 ago. 2021.

GÓMEZ JIMÉNEZ, Óscar; RODRÍGUEZ TORRES, Javier y CRUZ CRUZ, Purificación. Competencia digital del profesorado y la atención a la diversidad durante la COVID-19. Estudio de caso. Revista de Comunicación y Salud, v. 10, n. 2, p. 483-502, nov. 2020. DOI: 10.35669/rcys.2020.10(2).483-502. Disponible en: <http://www.revistadecomunicacionysalud.es/index.php/rcys/article/view/236>. Acceso en: 13 ago. 2021.

INSTITUTO NACIONAL DE TECNOLOGÍAS EDUCATIVAS Y DE FORMACIÓN DEL PROFESORADO (INTEF). Marco Común de Competencia Digital Docente. [S.I.: s.n.], 2017. Disponible en: <http://educalab.es/documents/10180/12809/MarcoComunCompeDigiDoceV2.pdf>. Acceso en: 13 ago. 2021.

JWA, Soomin. Modeling L2 Writer Voice: Discoursal Positioning in Fanfiction Writing. Computers and Composition, v. 29, n. 4, p. 323-340, dic. 2012. DOI: 10.1016/j.compcom.2012.10.001. Acceso en: 13 ago. 2021.

LÁZARO-CANTABRANA, José Luis; USART-RODRÍGUEZ, Mireia y GISBERT-CERVERA, Mercè. Assessing Teacher Digital Competence: the Construction of an Instrument for Measuring the Knowledge of Pre-Service Teachers. Journal of New Approaches in Educational Research, v. 8, n. 1, p. 73-78, ene. 2019. DOI: 10.7821/naer.2019.1.370. Disponible en: <https://naerjournal.ua.es/article/view/v8n1-10>. Acceso en: 13 ago. 2021.

MIRANDA LACERDA, Marcelo y SCHLEMMER, Eliane. Alfabetización digital, lengua y literatura en la cibercultura: los 'fanfics' como recurso pedagógico. Revista de Educación de la Universidad de Granada, v. 25, pág. 235, nov. 2018. DOI: 10.30827/reugra.v25i0.116. Disponible en:

<http://reugra.es/index.php/reugra/article/view/116>. Acceso en: 13 ago. 2021.

MULA-FRANCO, Antonio; LLORENS-GARCÍA, Ramón F. y ROVIRA-COLLADO, José. Didáctica lengua y literatura 2.0. Primeras experiencias TIC desde un máster de secundaria. In: ATAS do I Encontro Internacional TIC e Educação TICEDUCA2010. Lisboa: Instituto de Educação, 2010. p. 435-440. 
NIX, Ingrid y HALL, Marion. Collecting Questionnaire and Interview Data: Evaluating Approaches to Developing Digital Literacy Skills. 1 Oliver's Yard, 55 City Road, London EC1Y 1SP United Kingdom: SAGE Publications, Ltd., 2016. DOI: 10.4135/978144627305015595384. Disponible en:

<http://methods.sagepub.com/case/questionnaire-interview-evaluating-developing-digital-literacy-skills>. Acceso en: 13 ago. 2021.

ORTIZ COLÓN, Ana Maria; ÁGREDA MONTORO, Miriam y RODRÍGUEZ MORENO, Javier. Autopercepción del profesorado de Educación Primaria en servicio desde el modelo TPACK. Revista Electrónica Interuniversitaria de Formación del Profesorado, v. 23, n. 2, p. 53-65, abr. 2020. DOI: 10.6018/reifop.415641. Disponible en: <https://revistas.um.es/reifop/article/view/415641>. Acceso en: 13 ago. 2021.

PRENDES-ESPINOSA, María-Paz; CASTAÑEDA-QUINTERO, Linda y GUTIÉRREZ-PORLÁN, Isabel. Competencias para el uso de TIC de los futuros maestros. Comunicar: Revista Científica de Comunicación y Educación, v. 18, n. 35, p. 175-182, 2010. DOI: 10.3916/C35-2010-03-11. Disponible en:

$<$ https://www.revistacomunicar.com/index.php?contenido=detalles\&numero=35\&articulo=35-2010-21>. Acceso en: 13 ago. 2021.

REDECKER, Christine y PUNIE, Yves. European framework for the digital competence of educators (DigCompEdu). [S.I.: s.n.], 2017. Disponible en:

<https://publications.jrc.ec.europa.eu/repository/handle/JRC107466>. Acceso en: 13 ago. 2021.

ROVIRA COLLADO, José. Redes sociales de lectura: del libro de caras a la LIJ 2.0. Investigaciones Sobre Lectura, n. 3, p. 106-122, ene. 2015. DOI: 10.37132/isl.v0i3.36. Disponible en:

<http://www.comprensionlectora.es/revistaisl/index.php/revistalSL/article/view/36>. Acceso en: 13 ago. 2021.

ROVIRA-COLLADO, José y HERNÁNDEZ ORTEGA, José. Aspectos de literacidad digital en el desarrollo de los Entornos Personales de Aprendizaje (PLE) en el área de Lengua y Literatura. [S.I.]: Octaedro, 2016. Disponible en: <http://rua.ua.es/dspace/handle/10045/61841>. Acceso en: 13 ago. 2021.

RUIZ-DOMÍNGUEZ, M. M. y RODRÍGUEZ-MUÑOZ, F. J. Diseño y análisis del cuestionario "Conocimiento, uso y aceptación de recursos educativos tic en clase de literatura" aplicado a profesores de Educación Secundaria. In: DE AMO, J. M. (Ed.). La lectura y la escritura como prácticas sociales en la cultura digital. Valencia: Tirant lo Blanch, 2021. p. 163-186.

SÁ, María José y SERPA, Sandro. COVID-19 and the Promotion of Digital Competences in Education. Universal Journal of Educational Research, v. 8, n. 10, p. 4520-4528, oct. 2020. DOI:

10.13189/ujer.2020.081020. Disponible en: <http://www.hrpub.org/journals/article_info.php?aid=9799>. Acceso en: 13 ago. 2021.

SÁNCHEZ PRIETO, José Carlos; OLMOS MIGUELÁÑEZ, Susana y GARCÍA-PEÑALVO, Francisco J. Intención de uso de tecnologías mobiles entre los profesores en formación. Aplicación de un modelo de adopción tecnológica basado en TAM con los constructos compatibilidad y resistencia al cambio. In: RODRIGUES, M. R.; NISTAL, M. L. y FIGUEIREDO, M. (Ed.). Atas do XVII Simpósio Internacional de Informática Educativa (SIIE'15). Setúbal: Escola Superior de Educação do Instituto Politécnico de Setúbal, nov. 2015. p. 260-267. Disponible en: <https://repositorio.grial.eu/handle/grial/488>. Acceso en: 13 ago. 2021.

SCOLARI, Carlos (Ed.). Adolescentes, medios de comunicación y culturas colaborativas. Aprovechando las competencias transmedia de los jóvenes en el aula. Barcelona: Universitat Pompeu Fabra, 2018. Disponible en: <https://www.fundacionluminis.org.ar/biblioteca/adolescentes-medios-de-comunicacion-y-culturascolaborativas-aprovechando-las-competencias-transmedia-de-los-jovenes-en-el-aula $>$. Acceso en: 13 ago. 2021.

SUÁREZ RODRÍGUEZ, Jesús M.; ALMERICH, Gonzalo y col. Competencias del profesorado en las TIC. Influencia de factores personales y contextuales. Universitas Psychologica, v. 11, n. 1, p. 293-309, 2012. ISSN 2011-2777, 1657-9267. DOI: 10.11144/Javeriana.upsy11-1.cpif. Disponible en: <https://revistas.javeriana.edu.co/index.php/revPsycho/article/view/997>. Acceso en: 13 ago. 2021.

SUÁREZ RODRÍGUEZ, Jesús M.; ALMERICH CERVERÓ, Gonzalo y col. Las competencias en TIC del profesorado y su relación con el uso de los recursos tecnológicos. Archivos Analíticos de Políticas Educativas=Education Policy Analysis Archives, v. 18, n. 1, pág. 1, 2010. Disponible en: $<$ https://dialnet.unirioja.es/servlet/articulo?codigo=3199091>. Acceso en: 13 ago. 2021. 
TORRES, Ludwing. Hábitos de lectura, uso de internet y comprensión lectora. Un análisis de estudio de caso con estudiantes de secundaria del Colegio Saint Peter's. La Paz: Centro Psicopedagógico de Investigación en Educación Superior, Universidad Mayor de San Andrés, 2018.

VALERO GÓMEZ, Manuel y GRANADOS PÉREZ, Míriam. Educación, tecnología y alumnado. La lectura mediante las redes sociales. In: TECNOLOGÍA, innovación e investigación en los procesos de enseñanza-aprendizaje, 2016, ISBN 978-84-9921-848-9, págs. 1432-1440. [S.I.]: Octaedro, 2016.

p. 1432-1440. Disponible en: <https://dialnet.unirioja.es/servlet/articulo?codigo=6065799>. Acceso en: 13 ago. 2021.

\section{Contribuciones de los autores}

Francisco J. Rodríguez Muñoz: Investigación, Conceptualización, Metodología, Análisis formal, Redacción - Revisión y edición; María del Mar Ruiz Domínguez: Investigación, Conceptualización, Metodología, Análisis formal, Redacción - Revisión y edición . 